\title{
(2) OPEN ACCESS \\ Hyponatraemia despite isotonic maintenance fluid therapy: a time series intervention study
}

\author{
Milan Chromek, ${ }_{1}^{1,2}$ Åsa Jungner, ${ }^{1}$ Niclas Rudolfson, ${ }^{1}$ David Ley, ${ }^{1}$ Detlef Bockenhauer, ${ }^{3}$ \\ Lars Hagander (1) ${ }^{1}$
}

- Additional material is

published online only. To view please visit the journal online (http://dx.doi.org/10.1136/ archdischild-2019-318555).

${ }^{1}$ Paediatric Nephrology, Paediatric Surgery, Paediatric Intensive Care, and Neonata Care, Lund University, Skane University Hospital, Lund, Sweden

${ }^{2}$ Paediatric Nephrology, Karolinska Institute, Karolinska University Hospital, Stockholm, Sweden

${ }^{3}$ University College London, Department of Renal Medicine and Great Ormond Street Hospital for Children NHS Foundation Trust, London, UK

\section{Correspondence to} Lars Hagander, Department of Clinical Sciences Lund, Lund University, Lund 22185, Sweden;

lars.hagander@med.lu.se

Received 19 November 2019 Revised 16 September 2020 Accepted 19 September 2020 Published Online First 28 October 2020

\begin{abstract}
Objective To examine the prevalence of dysnatraemias among children admitted for paediatric surgery before and after a change from hypotonic to isotonic intravenous maintenance fluid therapy.

Design Retrospective consecutive time series intervention study.

Setting Paediatric surgery ward at the Children's Hospital in Lund, during a 7-year period, 2010-2017. Patients All children with a blood sodium concentration measurement during the study period were included. Hypotonic maintenance fluid $(40 \mathrm{mmol} / \mathrm{L} \mathrm{NaCl}$ and 20 $\mathrm{mmol} / \mathrm{L} \mathrm{KCl}$ ) was used during the first 3 years of the study (646 patients), and isotonic solution $(140 \mathrm{mmol} / \mathrm{L}$ $\mathrm{NaCl}$ and $20 \mathrm{mmol} / \mathrm{L} \mathrm{KCl}$ ) was used during the following period (807 patients).
\end{abstract}

Main outcome measures Primary outcomes were sodium concentration and occurrence of hyponatraemia ( $<135 \mathrm{mmol} / \mathrm{L}$ ) or hypernatraemia (>145 mmol/L). Results Overall, the change from hypotonic to isotonic intravenous maintenance fluid therapy was associated with a decreased prevalence of hyponatraemia from $29 \%$ to $22 \%$ (adjusted OR $0.65(0.51-0.82)$ ) without a significantly increased odds for hypernatraemia (from $3.4 \%$ to $4.3 \%$, adjusted OR $1.2(0.71-2.1)$ ). Hyponatraemia $<130 \mathrm{mmol} / \mathrm{L}$ decreased from $6.2 \%$ to $2.6 \%$, and hyponatraemia $<125 \mathrm{mmol} / \mathrm{L}$ decreased from $2.0 \%$ to $0.5 \%$.

Conclusions Routine use of intravenous isotonic maintenance fluids was associated with lower prevalence of hyponatraemia, although hyponatraemia still occurred in over $20 \%$ of patients. We propose that the composition and the volume of administered fluid need to be addressed.

\section{INTRODUCTION}

Intravenous solutions are used for rehydration and maintenance fluid therapy in children unable to receive oral fluids. Fluids can be classified according to their tonicity as either isotonic (approximately equal to plasma) or hypotonic (less than plasma). Since sodium constitutes the predominant effective osmole in plasma, the terms isotonic and hypotonic are commonly used with respect to the sodium concentration of the fluid. Previously, isotonic fluids were primarily used for acute rehydration, whereas maintenance fluids were designed as hypotonic solutions, to meet the daily requirements of fluid and electrolytes. ${ }^{1}$ Though conceptually reasonable, such hypotonic maintenance therapy has in clinical practice been associated with hyponatraemia and consequent risk for neurological sequelae and even

\section{What is already known on this topic?}

- Intravenous maintenance fluid therapy was originally designed as hypotonic.

- The use of hypotonic solutions has been associated with complications, including life-threatening hyponatraemia, and isotonic solutions are therefore currently recommended in paediatric clinical practice.

\section{What this study adds?}

- Isotonic maintenance fluid administration is associated with significantly lower prevalence of hyponatraemia without increasing odds for hypernatraemia.

- The prevalence of hyponatraemia is still high despite isotonic fluid maintenance.

death. ${ }^{2}$ The high incidence of hospital-acquired hyponatraemia is attributed to impaired free water secretion secondary to increased antidiuretic hormone $(\mathrm{ADH})$ secretion due to non-osmotic stimuli in acutely ill children. ${ }^{34}$ For these reasons, a cautious shift to isotonic solutions for maintenance therapy is increasingly advocated. ${ }^{56}$

The use of isotonic maintenance solutions may be associated with its own complications, for example, hypernatraemia or hyperchloraemic acidosis, and may be unsuitable for patients with renal concentrating defects or large extrarenal free water losses, and less appropriate for infants with a limited urine concentrating ability. ${ }^{5-13}$

The overnight change from hypotonic to isotonic maintenance fluid prescription (16 October 2013) in our paediatric surgery department offered a unique opportunity to evaluate the two different approaches. The purpose of this study was to assess the prevalence of hyponatraemia and hypernatraemia among children admitted for paediatric surgery before and after the introduction of isotonic intravenous maintenance fluid therapy.

\section{METHODS}

\section{Design and study population}

This is a retrospective consecutive time series intervention study of children admitted to the paediatric surgery ward at the Children's Hospital in Lund, a tertiary care university hospital, during a 7-year period, 2010-2017. The paediatric surgery ward at 
the Children's Hospital in Lund admits children with a weight $>2 \mathrm{~kg}$ and a postmenstrual age $>36$ weeks for paediatric surgery due to a wide spectrum of gastrointestinal and urogenital conditions, for both acute and elective surgeries. Included in the study were all children with at least one inpatient blood gas sodium concentration measurement.

\section{Primary exposure}

Two distinct study groups were formed based on exposure to hypotonic or isotonic maintenance solution. Hypotonic maintenance fluid (defined as $40 \mathrm{mmol} / \mathrm{L}$ sodium chloride and 20 $\mathrm{mmol} / \mathrm{L}$ potassium chloride in $5 \%$ glucose solution) was used during the first period (9 June 2010 to 15 October 2013). Isotonic solution (defined as $140 \mathrm{mmol} / \mathrm{L}$ sodium chloride and $20 \mathrm{mmol} / \mathrm{L}$ potassium chloride in 5\% glucose solution) was used during the second period (16 October 2013 to 17 December 2017). During both periods, the amount of intravenous maintenance fluid followed the Holliday-Segar formula and the European Society for Paediatric Gastroenterology, Hepatology, and Nutrition recommendations. ${ }^{12}$ During the entire study period, full maintenance rates for a 24-hour period were calculated as follows: bodyweight $\leq 10 \mathrm{~kg} 100 \mathrm{~mL} / \mathrm{kg} / 24$ hours, 10 to $\leq 20 \mathrm{~kg}$ $1000 \mathrm{~mL}+50 \times($ weight $(\mathrm{kg})-10) \mathrm{mL} / 24$ hours, $>20 \mathrm{~kg}, 1500$ $\mathrm{mL}+20 \times($ weight $(\mathrm{kg})-20) \mathrm{mL} / 24$ hours.

The study was blinded to the exact timing of blood samples and rates of infusion, but typically, children admitted for elective surgery started intravenous maintenance fluid treatment before surgery, with blood gas analyses postoperatively according to perceived clinical needs. The intraoperative fluid composition, the timing of fluid therapy and the practice of blood gas testing all remained unaltered throughout the study period, but on an individual level, the study was not able to differentiate between preoperative, perioperative and postoperative sodium values. No cases were excluded from analysis, and group assignment was based strictly on the cut-off date. In other words, the exposure in this study was based on an intention-to-treat policy, from which clinicians were free to deviate as deemed clinically appropriate. For example, the amount and composition of maintenance fluid could be individualised in rare cases of known renal failure and electrolyte disturbance. The scope of surgical care did not differ significantly during the study period (online supplemental figure 1).

\section{Primary outcome and independent variables}

The primary outcome was sodium concentration and prevalence of hyponatraemia and hypernatraemia. Sodium concentrations were retrieved from the hard drive of the two blood gas measurement machines (ABL 800, Radiometer, Copenhagen, Denmark) used during the study period. Hyponatraemia was defined as sodium concentration $<135 \mathrm{mmol} / \mathrm{L}$ and hypernatraemia as $>145 \mathrm{mmol} / \mathrm{L}$. Patient-level information on age and sex was derived from each patient's personal identification number.

\section{Statistical analysis}

The highest and the lowest measured sodium concentrations per patient are presented, as well as the presence of hyponatraemia and hypernatraemia, visualised per age. The $\chi^{2}$ test was used to compare the two exposure groups in terms of age and sex distribution, and the Mann-Whitney U test was used to compare the number of blood samples per patient. To compare the prevalence of dysnatraemia in the hypotonic and isotonic groups, we used multivariable logistic regressions with hypernatraemia or hyponatraemia as the dependent variable, study group as the
Table 1 The age and sex distribution of the hypotonic group and the isotonic group

\begin{tabular}{llll}
\hline & Hypotonic & Isotonic & P value \\
\hline $\mathrm{n}$ & 646 & 807 & \\
Number of tests (median (IQR)) & $2(1,5)$ & $2(1,4)$ & 0.07 \\
\hline Age (\%) & & & 0.2 \\
\hline 0-1 months & $106(16)$ & $168(21)$ & \\
\hline 1-6 months & $140(22)$ & $175(22)$ & \\
\hline 6-24 months & $119(18)$ & $129(16)$ & \\
$\quad$ Above 24 months & $281(44)$ & $335(42)$ & \\
Sex=male (\%) & $374(58)$ & $493(61)$ & 0.2 \\
\hline
\end{tabular}

independent variable, and with adjustment for age and sex. $\mathrm{P}$ values $<0.05$ were considered statistically significant. Data were analysed using the R software V.3.5.1 (Vienna, Austria).

\section{RESULTS}

\section{Patient characteristics}

A total of 7711 blood tests from 1453 patients were available for final analysis: 646 patients were included in the period when hypotonic maintenance fluids were recommended (hypotonic group) and 807 patients in the period with isotonic maintenance fluids (isotonic group) (online supplemental figure 2). The age and sex distribution of our cohorts is presented in table 1.

\section{The prevalence of hyponatraemia decreased but remained high after the introduction of isotonic maintenance fluid therapy}

First, we analysed the entire cohort, irrespective of age. After introduction of isotonic maintenance fluid therapy, the overall prevalence of hyponatraemia $(<135 \mathrm{mmol} / \mathrm{L})$ decreased from $190 / 646(29 \%)$ to $175 / 807$ (22\%), adjusted OR 0.65 (0.510.82 ) (figure 1 , all ages). The prevalence of hyponatraemia $<130 \mathrm{mmol} / \mathrm{L}$ decreased from $40 / 646(6.2 \%)$ to $21 / 807$ (2.6\%), adjusted OR $0.39(0.22-0.66)$, and the prevalence of hyponatraemia $<125 \mathrm{mmol} / \mathrm{L}$ decreased from $13 / 646$ (2.0\%) to $4 / 807$ $(0.5 \%)$, adjusted OR $0.24(0.08-0.73)$. The prevalence of hypernatraemia (>145 mmol/L) was not significantly different between the hypotonic group $(22 / 646(3.4 \%))$ and the isotonic group $(35 / 807(4.3 \%))$, adjusted OR $1.2(0.71-2.1)$. Very few children had both hyponatraemia and hypernatraemia during the same admission (1.4\%), and the range of sodium concentrations in individual patients did not significantly differ between the two time periods $(p=0.3)$. The regression models were adjusted for sex, which did not meaningfully change the results with regard to the main outcomes.

To assess the risk of dysnatraemia according to age, we analysed sodium levels with age as a continuous variable. In figure 2, the highest and lowest measured sodium levels per patient are depicted against the patients' age. Although the prevalence of dysnatraemias was higher in the neonatal group, fluid prescriptions in this age group were highly individualised, and dysnatraemias were deemed as secondary to events other than isotonic fluid administration. When analysing only children older than 1 month of age as a sensitivity analysis, the regression models confirmed a reduced odds for hyponatraemia (adjusted OR 0.61 $(0.46-0.80))$ after introduction of isotonic maintenance fluid administration, without increasing the odds of hypernatraemia (adjusted OR $0.70(0.35-1.4)$ ) (figure 1). 


$\begin{array}{ccccc}\text { Age group } & \text { Hypotonic period } & \text { Isotonic period } & \text { Hyponatraemia } & \text { OR } \\ \text { All ages } & 190 / 646(29 \%) & 175 / 807(22 \%) & -6.65[0.51-0.82] \cdots \\ & & & \\ >28 \text { days } & 152 / 540(28 \%) & 123 / 639(19 \%) & -6 & 0.61[0.46-0.80] \cdots\end{array}$

Hypernatraemia

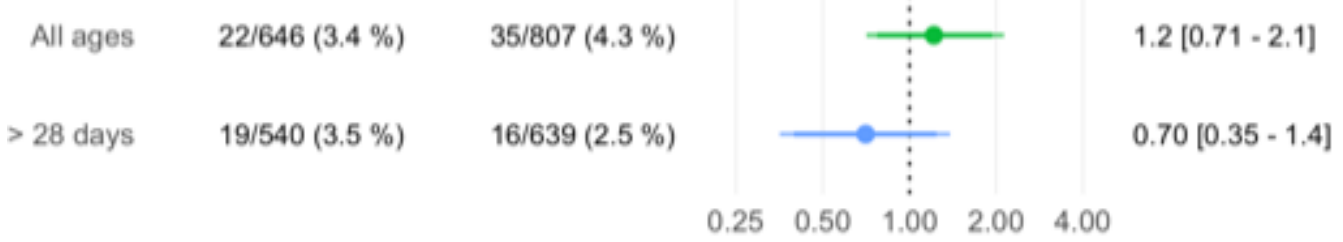

Odds Ratio

Figure 1 The prevalence (left) and odds ratio (right) for hyponatraemia (upper panel) and hypernatraemia (lower panel) are plotted according to administered fluid composition (isotonic period vs hypotonic period (reference). The odds for hyponatraemia decreased significantly but remained high, also in a sensitivity analysis of only the children older than 1 month ( $>28$ days).

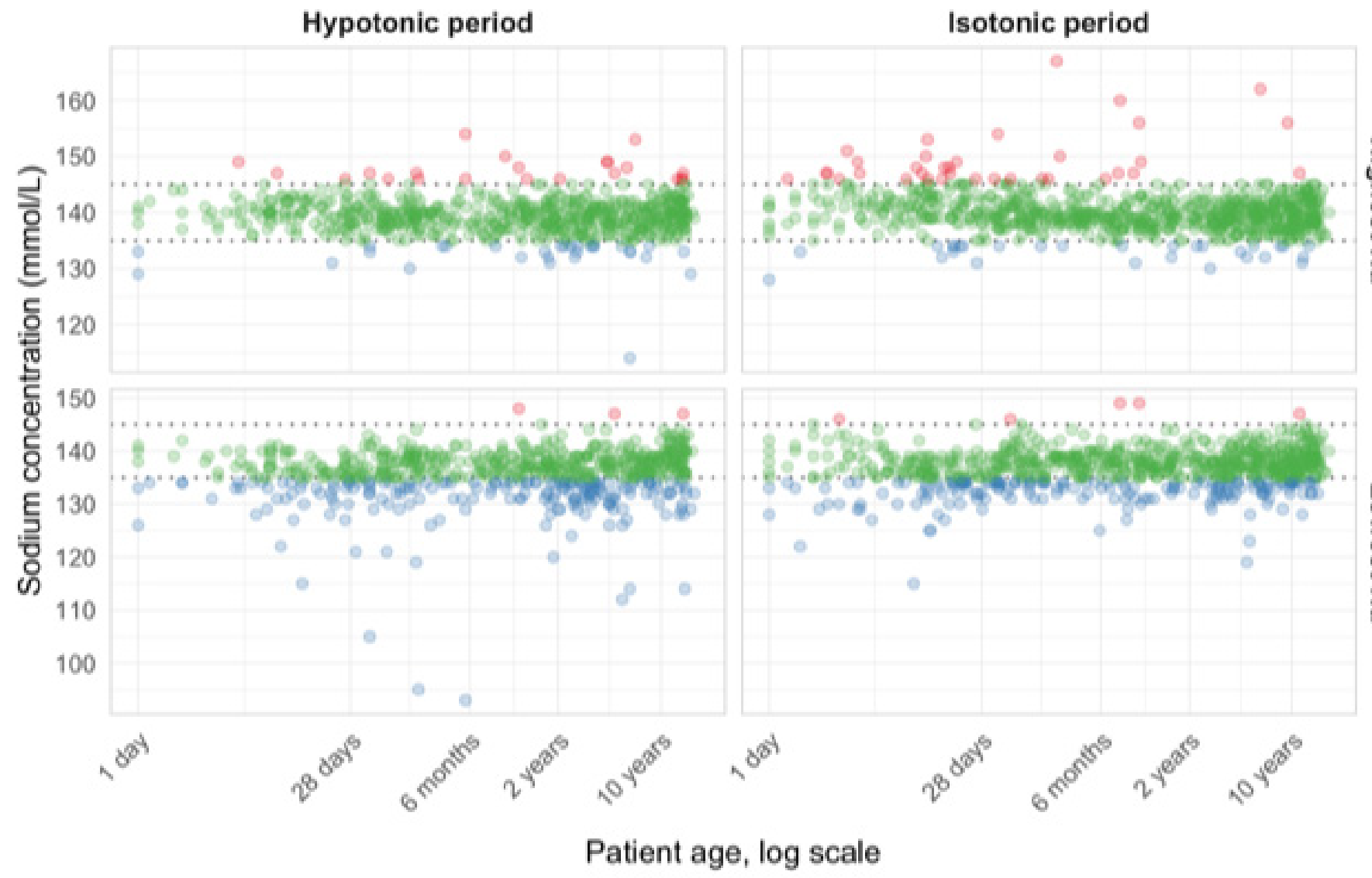

\section{Dysnatraemia • Hypernatraemia - Normal • Hyponatraemia}

Figure 2 Dysnatraemia during hospitalisation. Highest measured sodium concentrations per patient (upper panels) and lowest measured sodium concentrations per patient (lower panels) are plotted against age (0-18 years) in logarithmic scale. Panels on the left represent patients in the hypotonic group and panels on the right represent patients in the isotonic group. Values within the normal range (135-145 mmol/L) are plotted in green, values above the normal range in red and values below the normal range in blue. For neonates, the maintenance fluid administration was highly individualised, and hypernatraemia in this age group was most often secondary to events other than isotonic fluid administration. 


\section{DISCUSSION}

Maintenance fluids are used daily and worldwide, and the scientific evidence behind current clinical practice is still growing. Holliday and Segar in 1957 suggested a hypotonic composition of maintenance fluids based on basal daily requirements for water and electrolytes, ${ }^{1}$ and this practice was used for more than 50 years despite being associated with iatrogenic hyponatraemia. More than 100 cases of hyponatraemic encephalopathy with permanent neurological sequelae or even death have been reported. ${ }^{2} 14$ During the last 15 years, several studies found an association between hypotonicity of maintenance fluids and the development of hyponatraemia, ${ }^{15} 16$ and more than 15 randomised prospective trials have evaluated the safety and efficacy of isotonic fluids for the prevention of hyponatraemia. ${ }^{16} 17$ This resulted in new recommendations accepted by many centres including ours to use isotonic solutions for maintenance fluid therapy. 5618

In October 2013, the tonicity of standard maintenance fluids was changed overnight from hypotonic to isotonic at our tertiary paediatric surgery centre. This offered a unique opportunity to evaluate the effects of the introduced maintenance fluid strategy. To our knowledge, our cohort of children is the largest ever reported in this context. In line with previous studies and clinical trials, we found that perioperative isotonic maintenance fluid therapy was associated with lower prevalence of hyponatraemia without a corresponding significant increase in prevalence of hypernatraemia. Yet, importantly, even with isotonic maintenance, more than $20 \%$ of children were diagnosed with hyponatraemia. This highlights the difficulties of guidelines for fluid therapy to address the physiologic needs of all children. Our study includes children admitted for paediatric surgery, a group subjected to several non-osmotic stimuli for ADH secretion, such as pain, stress and certain medications. In this group of patients with increased levels of $\mathrm{ADH}$, hyponatraemia may develop although isotonic fluids have been administered. If the urine is more concentrated than the 'isotonic' fluid, then the administered fluid solution is actually hypotonic relative to urine and will thus result in excess free water administration. ${ }^{19}$ In our setting, the isotonic fluid prescribed had an effective osmolarity of $320 \mathrm{mOsm} / \mathrm{L}$ (140 mmol of $\mathrm{Na}, 20 \mathrm{mmol}$ of $\mathrm{K}$ and $160 \mathrm{mmol}$ of $\mathrm{Cl})$. Thus, excretion of these administered osmoles would result in a net gain of water, in the case of the patient's urine effective osmolarity (without urea) exceeding $320 \mathrm{mOsm} / \mathrm{L}$.

Our study excluded neonates from analysis of incidence of dysnatraemias. The reason was that in the majority of cases fluid treatment was individualised for neonates, and therefore the identified cases of neonatal dysnatraemia could not be attributed to isotonic maintenance fluid exposure. Water and sodium balance in neonates is more precarious due to several reasons, including a higher water content in the body, larger body surface area predisposing to higher insensible water losses and a limited ability to express and satisfy thirst. In addition, several aspects of renal function are immature in the neonates. Both glomerular filtration rate and tubular functions continue to develop during the first 2 years of postnatal development. The diluting ability of the neonatal kidneys is usually sufficient to maintain normal serum concentrations of sodium during intake of hypotonic breast milk. Conversely, urine concentration ability is significantly compromised, as it develops during the first year of life. ${ }^{91020-22}$ Because of the individualised fluid prescription in neonates in our study, no conclusions should be drawn regarding the treatment with isotonic or hypotonic fluids in this age group. Indeed, most studies on intravenous maintenance fluid therapy have excluded neonates, and the recently released clinical practice guidelines of the American Academy of Pediatrics are cautious about the use of isotonic maintenance fluids in neonates due to lack of data. ${ }^{6}$ Further studies are needed to determine the best standard maintenance solution in this age group.

Given that hyponatraemia in the context of intravenous fluid therapy is attributed to an excess of free water secondary to increased ADH secretion and not to a deficiency of sodium, ${ }^{23}$ infused fluid volume might need to be considered. So far, few studies have addressed the volume of maintenance fluid in addition to fluid tonicity for the prevention of hyponatraemia. A small study by Yung and Keeley suggested a lower prevalence of hyponatraemia when the maintenance fluid rate of hypotonic fluid was decreased by one-third $(p=0.12, n=50) .^{24}$ Similarly, a larger study by Kannan et al found a non-significant drop in the prevalence of hyponatraemia from $14 \%$ to $3.8 \%(p=0.056$, $\mathrm{n}=109$ ), when the amount of hypotonic maintenance fluid was reduced to two-thirds. ${ }^{25}$ Neville et al addressed the administration rate of both hypotonic and hypertonic maintenance fluids ( $n=31$ in each group). Although the reduction of hypotonic fluid volume did not influence the prevalence of hyponatraemia, the $50 \%$ reduction of isotonic fluid reduced the prevalence of hyponatraemia from $21 \%$ to $8 \%$, a non-significant finding. ${ }^{26}$ The statistical power of these studies is low and does not allow for any conclusions. However, considering these and our results, we believe that the tonicity and the volume of maintenance fluid should be addressed in a large controlled prospective randomised study.

Several previous studies have shown significant, but possibly clinically irrelevant differences between analysers using direct (blood gas analyser) and indirect (hospital laboratory autoanalyser) sodium measurements. The major source of disagreement was the variable concentration of plasma proteins causing pseudohyponatraemia or pseudonormonatraemia when the indirect electrodes were used, and these studies therefore favour the use of direct electrodes. ${ }^{27-29}$ Throughout the study, we used the same type of direct ion selective electrode analyser, which might be considered an advantage in terms of reliability and validity.

Our findings must be interpreted in the context of our study design. Despite the comprehensive inclusion of a large cohort in a mixed tertiary paediatric service, this remains a retrospective study, and while there was a clear pre-post exposure separation with an intention-to-treat policy and no known confounding factors changing synchronously, we cannot refute confounding with certainty. No other fluid administration aside from the maintenance fluids, for example, oral fluids or intravenous medications, has been considered in our analysis. Moreover, blood gas analyses were performed according to perceived clinical needs and not in a controlled research manner. Also, we provide data on plasma sodium concentrations, but do not have markers of kidney function, such as creatinine, as this is not measured on the blood gas analyser. We believe that acute or chronic kidney failure is an unlikely contributor to the observed prevalence of dysnatraemia in our cohort, since the vast majority of patients were admitted for gastrointestinal surgery and had no suspicion of underlying kidney disease, and since the rate of urological surgery remained constant. The question at hand in this study was the incidence of hospitalacquired dysnatraemia, yet as the study design was blind to the prevalence of preoperative hyponatraemia and hypernatraemia, we cannot claim that all reported dysnatraemia was a consequence of maintenance fluid administration. However, we can find no reason to suspect that the incidence of preoperative dysnatraemia would differ between the two exposure groups, and we therefore believe that the described differences 
between the two periods indeed can be attributed to changes in maintenance fluid protocol.

Despite the discussed limitations, the large number of patients included and the clear separation of administered hypotonic versus isotonic fluids due to the overnight change in maintenance fluid regime provide powerful evidence for a significantly but insufficient decreased odds for hyponatraemia with isotonic fluid administration.

Acknowledgements The authors would like to thank Ingela Andersson for excellent assistance in retrieving all blood gas analyses included in the study.

Contributors LH and MC conceptualised and designed the study, drafted the initial manuscript, and reviewed and revised the manuscript. ÅJ assisted with conceptualisation and design, and curated the data. NR collected the data, assisted on methodology design and carried out the initial analyses. DL assisted with conceptualisation and design. DB contributed with formal analysis. Å, NR, DL and DB critically reviewed the manuscript for important intellectual content.

Funding $M C$ received financial support from the Centre for Innovative Medicine (CIMED, Region Stockholm).

Competing interests None declared.

Patient consent for publication Not required.

Ethics approval Research ethics approval was obtained from the regional ethics committee in Lund, Sweden (diary number 2017/998).

Provenance and peer review Not commissioned; externally peer reviewed.

Data availability statement Data may be obtained from a third party and are not publicly available.

Supplemental material This content has been supplied by the author(s). It has not been vetted by BMJ Publishing Group Limited (BMJ) and may not have been peer-reviewed. Any opinions or recommendations discussed are solely those of the author(s) and are not endorsed by BMJ. BMJ disclaims all liability and responsibility arising from any reliance placed on the content. Where the content includes any translated material, BMJ does not warrant the accuracy and reliability of the translations (including but not limited to local regulations, clinical guidelines, terminology, drug names and drug dosages), and is not responsible for any error and/or omissions arising from translation and adaptation or otherwise.

Open access This is an open access article distributed in accordance with the Creative Commons Attribution Non Commercial (CC BY-NC 4.0) license, which permits others to distribute, remix, adapt, build upon this work non-commercially, and license their derivative works on different terms, provided the original work is properly cited, appropriate credit is given, any changes made indicated, and the use is non-commercial. See: http://creativecommons.org/licenses/by-nc/4.0/.

\section{ORCID iD}

Lars Hagander http://orcid.org/0000-0001-6407-4613

\section{REFERENCES}

1 Holliday MA, Segar WE. The maintenance need for water in parenteral fluid therapy. Pediatrics 1957;19:823-32.

2 Arieff $\mathrm{Al}$, Ayus JC, Fraser CL. Hyponatraemia and death or permanent brain damage in healthy children. BMJ 1992;304:1218-22.

3 Oh GJ, Sutherland SM. Perioperative fluid management and postoperative hyponatremia in children. Pediatr Nephrol 2016;31:53-60.

$4 \mathrm{McNab}$ S, Duke T, South M, et al. $140 \mathrm{mmol} / \mathrm{L}$ of sodium versus $77 \mathrm{mmol} / \mathrm{L}$ of sodium in maintenance intravenous fluid therapy for children in hospital (PIMS): a randomised controlled double-blind trial. Lancet 2015;385:1190-7.
5 Moritz ML, Ayus JC. Maintenance intravenous fluids in acutely ill patients. N Eng/ J Med 2015:373:1350-60

6 Feld LG, Neuspiel DR, Foster BA, et al. Clinical practice guideline: maintenance intravenous fluids in children. Pediatrics 2018:142:142.

7 Winberg J. The 24-hour true endogenous creatinine clearance in infants and children without renal disease. Acta Paediatr 1959;48:443-52.

8 Bockenhauer D, Zieg J. Electrolyte disorders. Clin Perinatol 2014;41:575-90.

9 Holtbäck U, Aperia AC. Molecular determinants of sodium and water balance during early human development. Semin Neonatol 2003;8:291-9.

10 Polacek E, Vocel J, Neugebauerova L, et al. The osmotic concentrating ability in healthy infants and children. Arch Dis Child 1965;40:291-5.

11 Hayes W. Ab-normal saline in abnormal kidney function: risks and alternatives. Pediatr Nephrol 2019;34:1191-9.

12 Guarino A, Ashkenazi S, Gendrel D, et al. European Society for pediatric gastroenterology, hepatology, and Nutrition/European Society for pediatric infectious diseases evidence-based guidelines for the management of acute gastroenteritis in children in Europe: update 2014. J Pediatr Gastroenterol Nutr 2014;59:132-52.

13 Bockenhauer D, Rees L, van't Hoff W. An association of tubular dysfunction, cortical macrocysts and chronic kidney disease. Pediatr Nephrol 2006;21:580-3.

14 Halberthal M, Halperin ML, Bohn D. Lesson of the week: acute hyponatraemia in children admitted to hospital: retrospective analysis of factors contributing to its development and resolution. BMJ 2001;322:780-2.

15 Carandang F, Anglemyer A, Longhurst CA, et al. Association between maintenance fluid tonicity and hospital-acquired hyponatremia. J Pediatr 2013;163:1646-51.

16 McNab S, Ware RS, Neville KA, et al. Isotonic versus hypotonic solutions for maintenance intravenous fluid administration in children. Cochrane Database Syst Rev 2014:CD009457.

17 Foster BA, Tom D, Hill V. Hypotonic versus isotonic fluids in hospitalized children: a systematic review and meta-analysis. J Pediatr 2014;165:163-9.

18 Moritz ML, Ayus JC. Prevention of hospital-acquired hyponatremia: a case for using isotonic saline. Pediatrics 2003:111:227-30.

19 Halperin ML, Skorecki KL. Interpretation of the urine electrolytes and osmolality in the regulation of body fluid tonicity. Am J Nephrol 1986;6:241-5.

20 Aperia A, Broberger $\mathrm{O}$, Thodenius $\mathrm{K}$, et al. Renal response to an oral sodium load in newborn full term infants. Acta Paediatr Scand 1972:61:670-6.

21 Rodríguez-Soriano J, Vallo A, Oliveros R, et al. Renal handling of sodium in premature and full-term neonates: a study using clearance methods during water diuresis. Pediatr Res 1983;17:1013-6.

22 Bonilla-Felix M. Development of water transport in the collecting duct. Am J Physiol Renal Physiol 2004;287:F1093-101.

23 Ellison DH, Berl T. Clinical practice. The syndrome of inappropriate antidiuresis. N Engl J Med 2007:356:2064-72.

24 Yung M, Keeley S. Randomised controlled trial of intravenous maintenance fluids. J Paediatr Child Health 2009:45:9-14.

25 Kannan L, Lodha R, Vivekanandhan S, et al. Intravenous fluid regimen and hyponatraemia among children: a randomized controlled trial. Pediatr Nephrol 2010;25:2303-9.

26 Neville KA, Sandeman DJ, Rubinstein A, et al. Prevention of hyponatremia during maintenance intravenous fluid administration: a prospective randomized study of fluid type versus fluid rate. J Pediatr 2010;156:313-9. e1-2.

27 Triplett KE, Wibrow BA, Norman R, et al. Can the blood gas analyser results be believed? A prospective multicentre study comparing haemoglobin, sodium and potassium measurements by blood gas analysers and laboratory auto-analysers. Anaesth Intensive Care 2019;47:120-7.

28 Dimeski G, Morgan TJ, Presneill JJ, et al. Disagreement between ion selective electrode direct and indirect sodium measurements: estimation of the problem in a tertiary referral hospital. J Crit Care 2012;27:326.e9-16.

29 Zhang JB, Lin J, Zhao XD. Analysis of bias in measurements of potassium, sodium and hemoglobin by an emergency department-based blood gas analyzer relative to hospital laboratory autoanalyzer results. PLOS One 2015;10:e0122383. 